\title{
HIGH ORDER MODE HEATING OBSERVATIONS IN THE PEP-II INTERACTION REGION*
}

\author{
Stan Ecklund, Franz-Josef Decker, Alan Fisher, Artem Kulikov, Mike Sullivan, SLAC, Stanford, \\ CA 94309, USA
}

Abstract

High order mode (HOM) heating is observed in the PEP-II [1] interaction region vacuum system by monitoring chamber temperatures in several locations. The region where the electron and positron beams enter a common chamber results in a RF cavity where the beams generate $\mathrm{HOM}$ heating. By determining the temperature dependence on beam current, beam bunch length, and phase of the two beams, information about the modes is extracted. The time response of the temperature and the geometry also aid in determining the source of the heat flow.

\section{INTERACTION REGION GEOMETRY}

The interaction region [2] vacuum chamber (Y chamber) brings the two ring vacuum chambers into a common chamber. This transition results in the cross sectional area seen by the beam to change abruptly, where the beam excites HOM locked in the created RF cavity. The interaction point (IP) has a vertex chamber $50 \mathrm{~mm}$ in diameter and $400 \mathrm{~mm}$ long. It is a double walled Be chamber with water cooling to remove induced HOM power. It is connected to a RF shielded bellows that connects to a larger B1/Q1 chamber made of Glidcop and copper. This larger aperture allows for the growing beam size and separation of the two beams as they go out from the interaction point. The B1/Q1 chamber between the Be vertex chamber and the point where the beams enter separate chambers is $2.4 \mathrm{~m}$ long and results in a cavity with trapped modes in the several $\mathrm{GHz}$ range. It also is designed to absorb synchrotron radiation with masks that necessitate bumps in the vacuum surface. The $\mathrm{Y}$ chamber on the downstream $\mathrm{e}^{-}$side of the IP has an abrupt vertical septum whereas the upstream side has a very gradual transition designed to intercept a higher power synchrotron fan.

The HOM heating was anticipated [3] in the construction of the interaction region vacuum chambers, and a specially cooled $\mathrm{SiC}$ absorber was incorporated into a second bellows section located $2 \mathrm{~m}$ from the interaction point. This lowers the $\mathrm{Q}$ of the resonant trapped modes and reduces the danger of exciting a narrow resonance to high fields.

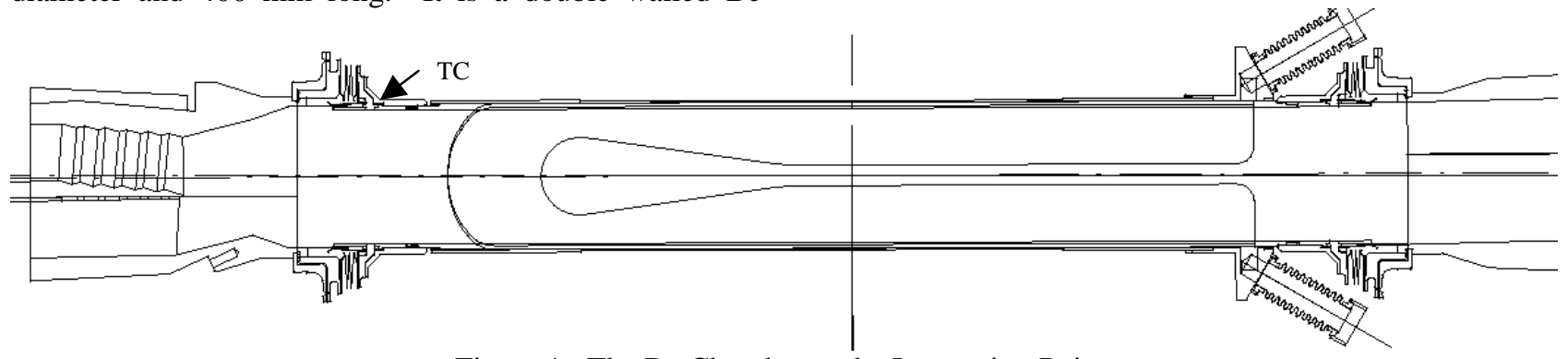

Figure 1: The Be Chamber at the Interaction Point.

\section{TEMPERATURE OBSERVATIONS}

Approximately 60 thermocouples (TC) were installed in the region within $2 \mathrm{~m}$ of the interaction point. They are used to monitor the cooling of synchrotron masks, vacuum chambers and magnets. One TC, called T_VTX2, located at the forward end of the Be chamber, near the bellows, shows higher readings than expected and causes concern about excessive heating in that region. It is in a region a few $\mathrm{cm}$ from the cooling channel on the $\mathrm{Be}$ chamber. With present beam currents of $0.8 \mathrm{~A}$ on 1.5 $\mathrm{A}$, it typically reads $150 \mathrm{~F}$, a rise of $90 \mathrm{~F}$ above the cooling water temperature. No RF probes were available in this region with the exception of a button beam position

\footnotetext{
* Work supported by US DOE contract DE-AC03-76-SF00515.
}

monitor $0.75 \mathrm{~m}$ from the IP. The signal is processed in the radiation area so wasn't initially available for examining the spectrum. The behavior of the TC reading was used to deduce the nature of the heating. A series of experiments were done changing conditions of the $\mathrm{e}^{-}$and $\mathrm{e}^{+}$beams.

\subsection{Thermocouple Time Response}

The TC response was found to respond slowly to changes in beam current. We found that a single exponential weighting of the history of the beam would approximately model the measured time response. Figure 2 shows the response to a rapid change in beam current caused by a beam abort. An exponential time constant of 220 seconds was consistently found for the T_VTX2 TC (TC in figure 1). 


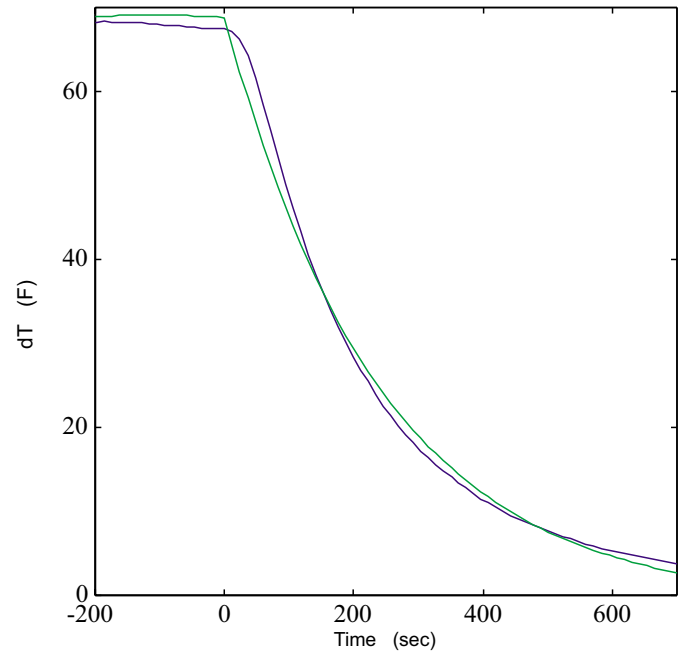

Figure 2: Response of thermocouple (blue) and exponential weighting from positron current (green) for a step change of $-1.25 \mathrm{~A}$.

\subsection{Current Dependence}

The current dependence could be extracted by fitting the TC readings to linear $(\mathrm{H}, \mathrm{L})$ and quadratic terms $(\mathrm{M}, \mathrm{N})$ in each beam, using the exponential time weighting of the currents. The time weighting allows one to take data more quickly since it accounts for the slow response of the TC.

$\mathrm{T}=\mathrm{T}_{0}+\mathrm{HI}_{-}+\mathrm{LI}_{+}+\mathrm{MI}_{-}^{2} / \mathrm{N}_{\mathrm{b}}+\mathrm{NI}_{+}^{2} / \mathrm{N}_{\mathrm{b}}+\mathrm{aI} \mathrm{I}_{+} / \mathrm{N}_{\mathrm{b}}$
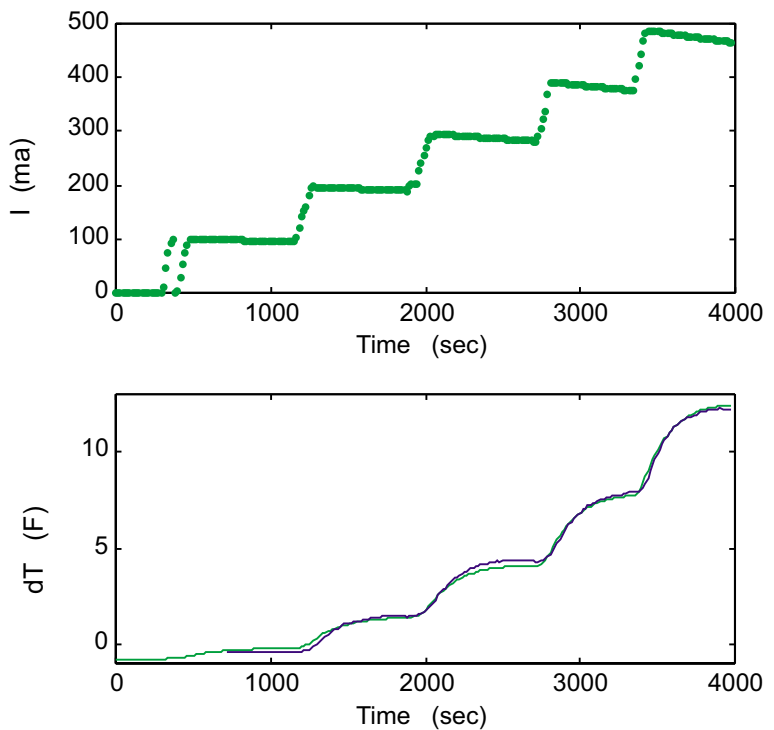

Figure 3: Step increases of the positron current (top). The bottom graph shows measured Temperature rise (blue) and fit (green) based on square of positron current and response time.
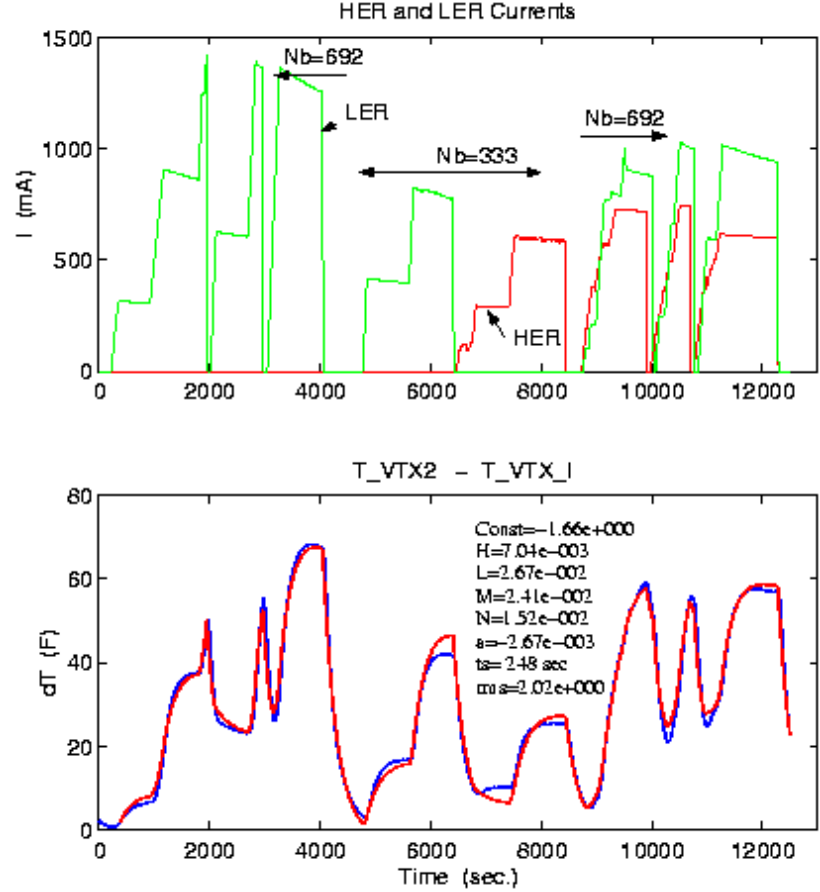

Figure 4: Data (blue) with changing electron and positron currents and fit (red).

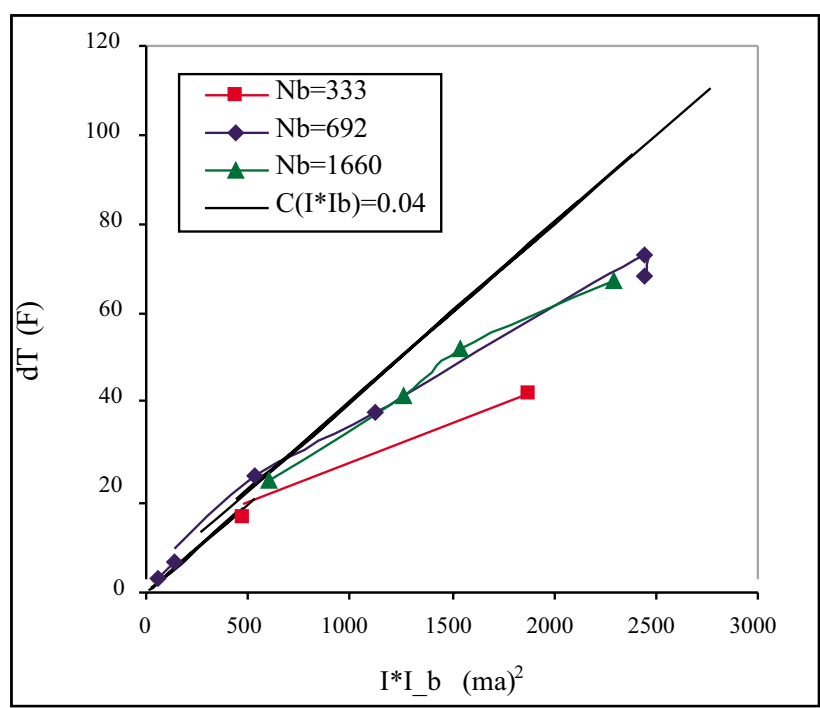

Figure 5: Temperature rise vs. product of bunch current times average current for various bunch fill patterns denoted by the number of populated bunches $(\mathrm{Nb})$. The line shows the expected behavior from low currents.

At low currents we find a quadratic dependence on current, or specifically a temperature rise proportional to the product of bunch current times average current, as expected for HOM heating. At higher currents, we found both linear and quadratic terms are required, implying a change in the beam bunch length, or nonlinear cooling from radiation or convection. 


\subsection{Dependence on $R F$ voltage}

To investigate the dependence upon beam bunch length, a series of runs were taken changing the RF total gap voltage in the LER (positron beam). The bunch length is expected to vary as the inverse square root of the gap voltage and the HOM power to increase with shorter bunch size. The data show a strong dependence but are not sufficient to check the expected functional dependence.

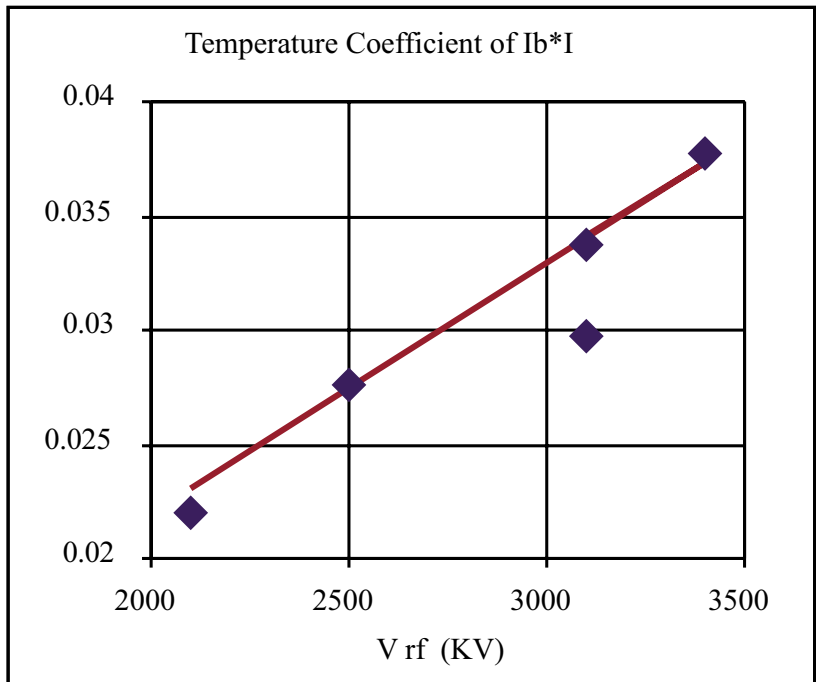

Figure 6: Dependence of HOM power on RF gap voltage determined from the fit coefficient of ( $\left.I_{\text {ave }} I_{\text {bunch }}\right)$.

\subsection{Two Beam Interference}

We wanted to determine if any single HOM resonance was responsible for the heating seen. Since both beams excite HOM heating seen by this TC, we could determine if the excitation by the two beams was coherent. We put a fixed current in each ring of $700 \mathrm{~mA}$ electrons in the HER and $1000 \mathrm{~mA}$ positrons in the LER. The RF phase of the HER was then moved relative to the LER. The beams were separated vertically so any effects of moving in and out of collision longitudinally were avoided. The change in temperature from nominal divided by the product of the two bunch currents times number of bunches was used to determine a normalized excitation. We found a modulation amplitude of $0.0034 \mathrm{deg} . \mathrm{F} / \mathrm{mA}^{2}$ or about $10 \%$ of the nominal heating.

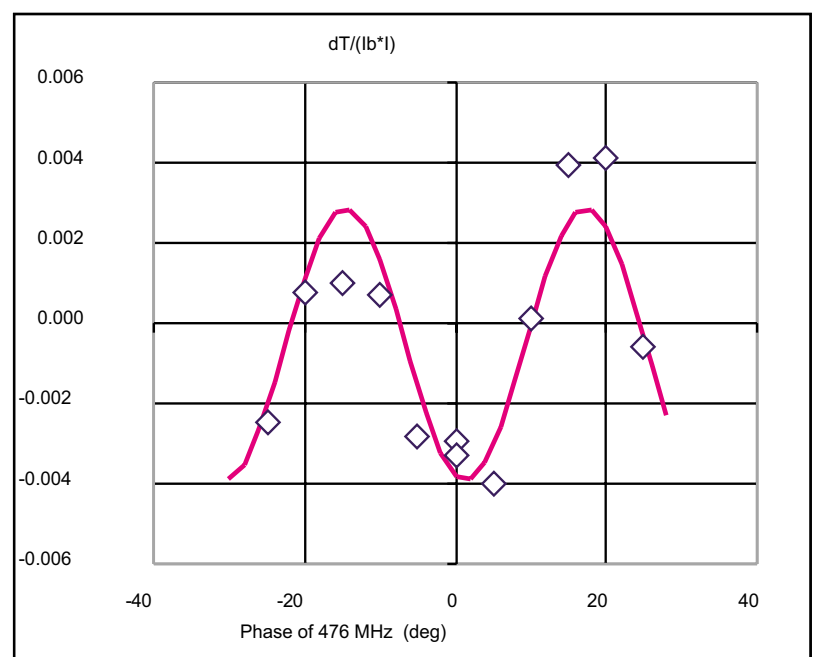

Figure 7: Modulation of heating by both beams changing the phase of the electron beam. The curve corresponds to $5.4 \mathrm{GHz}$

\subsection{Heating at other locations}

We have focused here on the behavior of a particular thermocouple where heating is a concern. Others provide information as well. One located in a similar place, but on the incoming e- side of the interaction point behaves identically except the temperature rise is $1 / 3$ as large.

Thermocouples on the water out pipes and knowledge of the flow rates give an estimate of the power removed with $20 \%$ accuracy. For the full currents noted above we estimate $600 \mathrm{~W}$ is being removed from the vertex Be chamber. The outboard bellows with $\mathrm{SiC}$ absorber at $2 \mathrm{~m}$ from the IP dissipates $5 \mathrm{~kW}$ on the outgoing $\mathrm{e}^{-}$side and less than half that amount on the incoming $\mathrm{e}^{-}$side. We expect this difference is due to the difference in the $\mathrm{Y}$ chamber transitions.

\section{CONCLUSION}

With simple temperature measurements, we have been able to determine the nature of heating in a particular region of the interaction region vacuum system. The current dependence identifies HOM as the source of heat. The response to RF voltage shows the expected bunch length sensitivity. Interference between the two beams is found to be at the $10 \%$ level. Understanding the time response of the system was important to analyzing the data with minimal bias. HOM calculations and thermo modeling of the system are underway.

\section{REFERENCES}

[1] "PEP-II An Asymmetric B Factory Conceptual Design Report." SLAC-418, June 1993.

[2] M. Sullivan, "Beam-beam collisions at the PEP-II B Factory," PAC 99, Mar. 1999.

[3] X.Lin et.al., "HOM heating at the PEP-II B-factory IR beryllium vacuum pipe," PAC 97, Vancouver, May 1997. 